\title{
Near-Eddington Luminosity Flares from Quiescent Supermassive Black Holes
}

\author{
Suvi Gezari ${ }^{1,2}$ \\ ${ }^{1}$ Department of Physics and Astronomy, Johns Hopkins University, \\ 3400 North Charles St., Baltimore, MD 21218, USA \\ Email: suvi@pha.jhu.edu \\ ${ }^{2}$ Hubble Fellow
}

\begin{abstract}
A dormant supermassive black hole lurking in the center of a galaxy will be revealed when a star passes within its tidal disruption radius, is disrupted, and a flare of electromagnetic radiation is emitted when the bound stellar debris is accreted. Although the tidal disruption of a star is a rare event in a galaxy, $\sim 10^{-4} \mathrm{yr}^{-1}$, observational candidates have emerged in all-sky X-ray and deep UV surveys in the form of luminous UV/X-ray flares from otherwise quiescent galaxies. We present the light curves and broadband properties of three tidal disruption candidates discovered in the UV by GALEX, and find that (1) the light curves are well-fitted by the power-law decline expected for the fallback of debris from a tidally disrupted solar-type star, and (2) the UV/optical spectral energy distributions can be attributed to thermal emission from an envelope of debris located at ten times the tidal disruption radius of the central black hole. We use the observed peak absolute optical magnitudes of the flares to predict the detection capabilities of the next generation of wide-field optical synoptic surveys.
\end{abstract}

Keywords. galaxies: nuclei, ultraviolet: galaxies, surveys, accretion

\section{Introduction}

Stellar dynamical models predict that once every $10^{3}-10^{5}$ yr a star in a galaxy will pass within the tidal disruption radius of the central black hole, $R_{p}<R_{T}=R_{\odot}\left(M_{\mathrm{BH}} / M_{\odot}\right)^{1 / 3}$, and will be torn apart by tidal forces (Magorrian \& Tremaine 1999; Wang \& Merritt 2004). After the star is disrupted, at least half of the debris is ejected from the system, and some fraction, $(0.1-0.5) M_{\odot}$, remains bound to the black hole and is accreted (Rees 1988; Ayal et al. 2000). The fallback of debris onto the black hole, and stream-stream collisions between precessing debris orbits (Rees 1988; Cannizzo et al. 1990; Kim et al. 1999) produce a luminous electromagnetic flare that is expected to peak in the UV/Xrays (Ulmer 1999).

Detections of tidal disruption events are of interest because they are a signpost for the presence of a dormant supermassive black hole in the nucleus of a galaxy that is otherwise quiescent and undetectable (Frank \& Rees 1976; Lidskii \& Ozernoi 1979; Rees 1988). The observed properties of tidal disruption flares have the potential to probe the mass and spin of supermassive black holes in distant galaxies, independently of locally established scaling relations between the mass of the central black hole and its global host galaxy properties. Tidal disruption events may also reveal the presence of intermediate mass black holes in globular clusters and dwarf galaxies (Ramirez-Ruiz \& Rosswog 2008). However, for the most massive black holes, the tidal disruption radius is smaller than the event horizon and stars are swallowed whole without disruption (for a solar-type star $M_{\text {crit }} \gtrsim 10^{8} M_{\odot}$, Hills 1975$)$. 
The most convincing candidates for a tidal disruption event are luminous, UV/X-ray flares from galaxies which have no ongoing AGN activity for which a flare could be attributed to the AGN's central engine. Strong candidates have now emerged in all-sky X-ray surveys (ROSAT All-Sky Survey, Donley et al. 2002; XMM-Newton Slew Survey, Esquej et al. 2007) and in the deep ultraviolet (UV) GALEX Deep Imaging Survey (DIS) (Gezari et al. 2006, 2008, 2009).

\section{Tidal Disruption Event Candidates Discovered by GALEX}

In a systematic search of the GALEX Deep Imaging Survey (DIS) in the FUV and NUV, we discovered luminous UV flares from three non-active galaxies: two early-type galaxies (D1-9 at $z=0.326$ and D3-13 at $z=0.3698$ ) and one star-forming galaxy (D23H-1 at $z=0.1855$ ). In follow-up Chandra imaging, two of the flares were detected only at extremely soft X-ray energies (D1-9 and D3-13) and one was not detected at all (D23H-1). Because some of the DIS fields overlap with the optical CFHT Legacy Survey (CFHTLS) Deep Survey, we were able to extract simultaneous optical difference imaging light curves of D1-9 and D3-13 in the $g, r$, and $i$ bands with an excellent cadence of days. In the case of D23H-1, we obtained TOO imaging with the Palomar LFC in the $g$ band.

The UV and optical light curves of the events are in excellent agreement with the theoretical expectations for emission from a tidally disrupted main sequence star. In Figure 1 we show a fit to the analytical equation from Lodato et al. (2009) for the mass accretion rate of a tidally disrupted solar-type star with a polytropic index, $\gamma=1.4$, which takes into account the internal structure of the star in the calculation. We take their equation for $\dot{M}(t)$ for a solar-type star disrupted by a $10^{6} M_{\odot}$ black hole at $R_{p}=R_{T}$, and scale it by $k^{-3 / 2} M_{6}^{1 / 2}(1+z)$, where $M_{6}=M_{\mathrm{BH}} / 10^{6} M_{\odot}$ and $k$ is the spin-up parameter. Assuming the star is spun-up to near break up upon disruption and thus $k=3$, the fits to the L09 curve have $M_{\mathrm{BH}}=5.4 \times 10^{7}, M_{\mathrm{BH}}=2.4 \times 10^{7} M_{\odot}$, and $M_{\mathrm{BH}}=1.2 \times 10^{7} M_{\odot}$ and for D23H-1, D1-9, and D3-13, respectively. In Figure 2, we show the broadband spectral energy distributions (SEDs) of the flares fitted with a single temperature blackbody of $T_{\mathrm{bb}}=1.7 \times 10^{5}, 5.6 \times 10^{4}$, and $4.9 \times 10^{4} \mathrm{~K}$, for D23H-1, D1-9, and D3-13, respectively, corresponding to peak bolometric luminosities of $\log L_{\mathrm{bol}}\left(\operatorname{ergs~s}^{-1}\right)=45.5,43.6$, and 43.8 .

\section{Demographics of Tidal Disruption Event Candidates}

Now that the census of tidal disruption candidates has reached a total of 10 , we can start to compare their properties and investigate the selection effects that arise from their different modes of discovery. The ROSAT All-Sky Survey (RASS) detected large amplitude, luminous soft X-ray outbursts from four galaxies with no previous evidence of AGN activity. The soft X-ray flares were well described by blackbody spectra with $T_{\mathrm{bb}}=$ $(6-12) \times 10^{5}$ K (Bade et al. 1996; Komossa \& Bade 1999; Komossa \& Greiner 1999; Grupe et al. 2003; Greiner et al. 2000). More recently a flaring soft X-ray source was reported from a member of the cluster Abell 3571 with a peak luminosity of $L(0.3-2.4 \mathrm{keV})=$ $6.8 \times 10^{42} \mathrm{ergs} \mathrm{s}^{-1}$ and blackbody temperature of $k T_{\mathrm{bb}}=0.12 \mathrm{keV}$, that decayed by a factor of $>650$ over $10 \mathrm{yr}$ (Cappelluti et al. 2009). The XMM Slew Survey (XMMSL1) detected large amplitude soft X-ray flares from two galaxies with no previously known AGN. Only one of the flares had enough counts to be directly fitted with a blackbody with $T_{\mathrm{bb}}=1.1 \times 10^{6} \mathrm{~K}$, while the other had counts only in the $0.2-2 \mathrm{keV}$ band, and was assumed to have $T_{\mathrm{bb}}=8 \times 10^{5} \mathrm{~K}$ (Esquej et al. 2008). A UV flare detected by HST/FOC in the nucleus of NGC 4552 (Renzini et al. 1995; Cappellari et al. 1999) was 


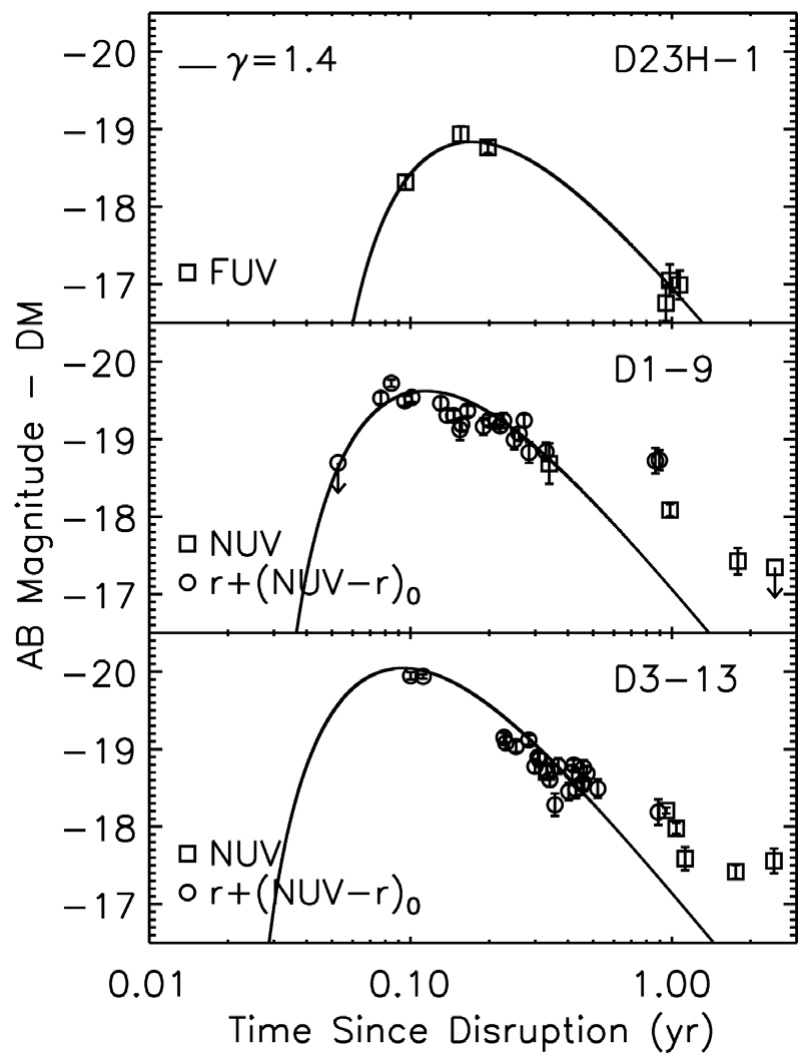

Figure 1. Light curves of the three GALEX tidal disruption flare candidates, presented in Gezari et al. (2006, 2008, 2009). The AB magnitudes have been corrected for Galactic exctinction, and subtracted by the distance modulus $\mathrm{DM}=5 \log \left(d_{\mathrm{L}} / 10 \mathrm{pc}\right)$. The time axis is in years since the time of disruption found in the fit, corrected for time dilation, in the rest-frame of the galaxy. The solid line shows the analytical solution from Lodato et al. (2009) of $\dot{M}(t)$ for a solar-type star with a $\gamma=1.4$ polytropic index, scaled in time by $k^{-3 / 2}\left(M_{\mathrm{BH}} / 10^{6} M_{\odot}\right)^{1 / 2}$.

interpreted as the accretion of a tidally stripped stellar atmosphere by the mini-AGN at its center. However, this galaxy was shown to have another episode of variability $10 \mathrm{yr}$ later, which casts doubt on this interpretation (Maoz et al. 2005). If we interpret the steep power-law shapes of the UV and soft X-ray candidates as the Rayleigh-Jeans and Wien tail of a thermal blackbody spectrum, respectively, then their blackbody temperatures, in combination with their bolometric luminosities can be used to infer a characteristic radius of the emission. Figure 3 shows the peak bolometric luminosity and blackbody spectrum temperature $\left(k T_{\mathrm{bb}}\right)$ of the four candidates from RASS summarized in Komossa et al. (2002), the Abell cluster candidate from Cappelluti et al. (2009), two candidates from XMMSL1 reported by Esquej et al. (2007, 2008), and the three candidates discovered by GALEX reported by Gezari et al. $(2006,2008,2009)$. The blackbody component of D23H-1 is shown both without the internal extinction correction (solid symbol) and with a correction for $E(B-V)_{\text {gas }}=0.3$ (open symbol), and connected with a solid line. For the GALEX candidates that had a soft X-ray detection during their flare, we connect the UV/optical blackbody component to the soft X-ray blackbody component (plotted with an X) with a dotted line. Because the X-ray detections for these candidates were $\sim 1-2$ years after the peaks of the flares, we scale the fluxes using the light curve fits to estimate their X-ray luminosities during the peak. However, this is highly uncertain, 


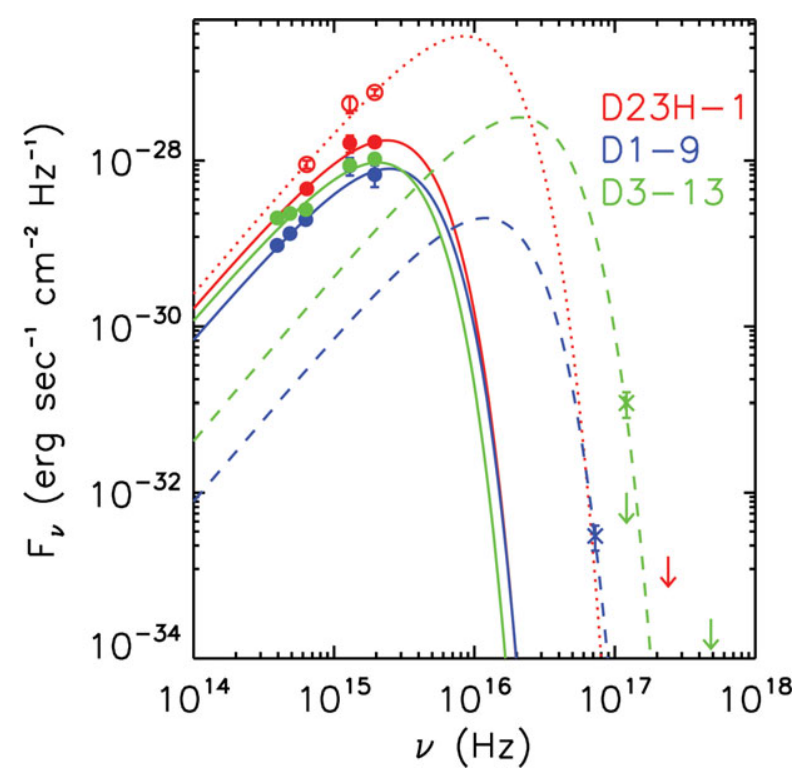

Figure 2. Single temperature blackbody fits to the 3 GALEX flares. Fits to the soft X-ray flux densities in D1-9 and D3-13 are shown with a dashed line and were detected 2.3 and 1.2 yr after the peak of their flares, respectively. Fluxes corrected for an internal extinction of $E(B-V)_{\text {gas }}=0.3$ using the Calzetti (2001) extinction law are plotted with open symbols, and the corresponding blackbody fit is plotted with a dotted line.

since the X-ray detection for D3-13 showed variability of a factor of 10 on a one-day timescale, and may not follow the decay of the UV/optical light curve. We plot the soft X-ray component of D3-13 as an upper limit to account for this variability.

The black hole masses inferred from the host galaxies of the candidates using locally established scaling relations between $M_{\mathrm{BH}}$ and the host galaxy bulge, yield black hole masses for the XMMLSS1 candidates of a few times $10^{6} M_{\odot}, \sim 10^{7} M_{\odot}$ for the Abell cluster candidate (Cappelluti et al. 2009), and a few times $10^{7} M_{\odot}$ for the GALEX candidates and the ROSAT candidate NGC 5905. The inferred radii for the X-ray selected candidates are much smaller than $R_{T}$ for these black hole masses, and may be associated with the innermost regions of the accretion torus. In contrast, the blackbody radii inferred for the UV/optical component of the GALEX flares are 10 times the tidal disruption radius of a $10^{7} M_{\odot}$ black hole, shown with a dotted line in Figure 3 . The presence of material at such large radii is a natural consequence of the disruption of a star, since at least half of the stellar mass is unbound at high velocities during the disruption process. An extended envelope of debris may form and reprocess the radiation from the inner accretion torus with an effective temperature of $T_{\text {eff }} \approx 2.3 \times 10^{4} \mathrm{~K}\left(M_{\mathrm{BH}} / 10^{7} M_{\odot}\right)^{1 / 4}($ Loeb \& Ulmer 1997; Ulmer et al. 1998), close to the temperatures fitted to the UV/optical components of the GALEX flares.

\section{Conclusions}

The next generation of wide-field synoptic surveys will be at optical wavelengths, and their capabilities for detecting tidal disruption events will depend on how these flares manifest themselves in this wavelength range. We have now successfully detected 3 candidates for tidal disruption events from quiescent SMBHs in the optical, and we can estimate the detection rate of future optical surveys by using the observed range of 
Galactic extinction-corrected peak absolute $g$-band magnitudes for the GALEX candidates, $M_{g}(\mathrm{D} 1-9)=-17.66 \pm 0.05, M_{g}(\mathrm{D} 3-13)=-18.88 \pm 0.05, M_{g}(\mathrm{D} 23 \mathrm{H}-1)=$ $-17.52 \pm 0.12$ mag. We estimate the black-hole mass-dependent volume rate as $N\left(M_{\mathrm{BH}}\right)=$ $\dot{N}\left(M_{\mathrm{BH}}\right) N_{\mathrm{BH}}\left(M_{\mathrm{BH}}\right) f\left(M_{\mathrm{BH}}\right)$, where $\dot{N}\left(M_{\mathrm{BH}}\right)$ is the tidal disruption rate as a function of black hole mass found for elliptical galaxies by Wang \& Merritt (2004), $\dot{N}\left(M_{\mathrm{BH}}\right) \sim$ $1.6 \times 10^{-4} \mathrm{yr}^{-1} M_{6}^{-0.3}, N_{\mathrm{BH}}$ is the black hole mass function estimated from the Ferguson \& Sandage (1991) E+S0 luminosity function scaled to black hole mass using the $M_{\mathrm{BH}^{-}}$ $M_{\text {bulge }}$ relation from Merritt \& Ferrarese (2001) and the mean galaxy mass-to-light ratio from Magorrian et al. (1998) with a factor of two to account for the bulges of spiral galaxies, and $f\left(M_{\mathrm{BH}}\right)$ is the fraction of flares that have a mass accretion rate close to the Eddington limit for a Salpeter mass function from Ulmer (1999). If we integrate $\dot{N}\left(M_{\mathrm{BH}}\right)$ over the full range of black hole masses, $10^{7}-10^{8} M_{\odot}$, this yields $N=1.3 \times 10^{-5} \mathrm{yr}^{-1}$ $\mathrm{Mpc}^{-3}$. For this volume rate, the $50 \mathrm{deg}^{2}$ Pan-STARRS1 Medium Deep Survey (PS1 MDS) will detect 32 events $\mathrm{yr}^{-1}$ with $g<22$ mag out to $790 \mathrm{Mpc}$ (or $z=0.16$ ).

We have chosen this brightness cut off so that the peak of the flares is at least 2 mag brighter than the detection limit of the survey to allow for the decay of the light curve of the flare to be followed in detail. However, here we have assumed that all flares will have a peak with $M_{g}<-17.5$. If instead we take into account that these luminosities apply only to the higher-mass black holes, i.e., $M_{\mathrm{BH}}>10^{7} M_{\odot}$, then the volume rate for this black hole mass range, $2.3 \times 10^{-6} \mathrm{yr}^{-1} \mathrm{Mpc}^{-3}$, corresponds to a detection rate for PS1 MDS of $5 \mathrm{yr}^{-1}$. This can be thought of as a lower limit, since the survey will be sensitive to fainter events from lower-mass black holes at smaller distances. The future Large Synoptic Survey Telescope (LSST) will act as a tidal disruption event factory, since

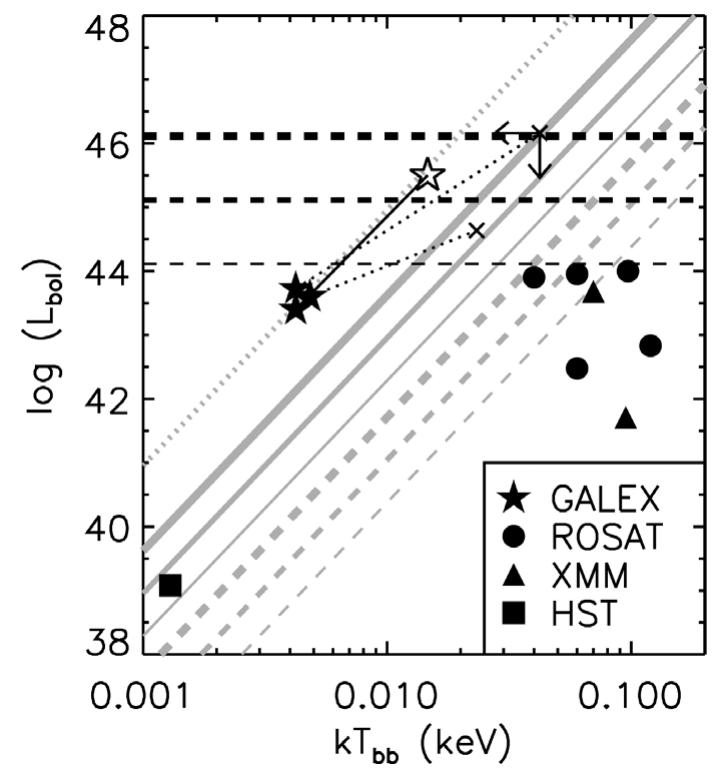

Figure 3. Peak bolometric luminosity of candidate tidal disruption events as a function of their blackbody temperature. GALEX candidates with soft X-ray detections during their flares have the estimated peak soft X-ray blackbody component plotted with an X. Solid grey lines show the luminosity as a function of temperature for a blackbody radius equal to the tidal disruption radius of a $10^{6}, 10^{7}$, and $10^{8} M_{\odot}$ black hole, and dotted grey line shows the luminosity for blackbody radius equal to ten times the radius of a $10^{7} M_{\odot}$ black hole. Dashed grey lines show the same relation, but for $T_{\mathrm{bb}} / T_{\mathrm{eff}}=3$. Dashed black lines show the Eddington luminosity of a $10^{6}, 10^{7}$, and $10^{8} M_{\odot}$ black hole. 
its depth and cadence will be similar to PS1 MDS, but with a factor of 400 increase in area that will increase the detection rates by two orders of magnitude!

\section{References}

Ayal, S., Livio, M., \& Piran, T. 2000, ApJ, 545,772

Bade, N., Komossa, S., \& Dahlem, M. 1996, A\& A, 309, L35

Cannizzo, J. K., Lee, H. M., \& Goodman, J. 1990, ApJ, 351, 38

Cappelluti, N., et al. 2009, A\&A, 495, L9

Donley, J. L., Brandt, W. N., Eracleous, M. J., \& Boller, Th. 2002, AJ, 124, 1308

Esquej, R. D., et al. 2006, A\& $A, 462$, L49

Esquej, R. D., et al. 2008, A\&BA, 489, 543

Frank, J. \& Rees, M. J. 1976, MNRAS, 176, 633

Gezari, S., et al. 2006, ApJ, 653, L25

Gezari, S., et al. 2008, ApJ, 676, 944

Gezari, S., et al. 2009, ApJ, 698, 1367

Greiner, J., Schwarz, R., Zharikov, S., \& Orio, M. 2000, A\& A, 362, L25

Grupe, D., Thomas, H. C., \& Leighly, K. M. 2003, ApJ, 592, 42 (erratum 2004, ApJ, 601, 1159)

Hills, J. G. 1975, Nature, 254, 295

Kim, S. S., Park, M.-G., \& Lee, H. M. 1999, ApJ, 519, 647

Komossa, S. 2002, in Lighthouses of the Universe, ed. M. Gilfanov, R. A. Sunyaev, \& E. Churazov (Berlin: Springer), 436

Komossa, S., et al. 2004, ApJ, 603, L17

Komossa, S. \& Bade, N. 1999, A\&A, 343, 775

Komossa, S. \& Greiner, J. 1999, A\&AA, 349, L45

Li, L-X., Narayan, R., \& Menou, K. 2002, ApJ, 576, 753

Lidskii, V. V., \& Ozernoi, L. M. 1979, Soviet Astron. Lett., 5, 16

Lodato, G., King, A. R., \& Pringle, J. E. 2009, MNRAS, 392, 332

Loeb, A. \& Ulmer, A. 1997, ApJ, 489, 573

Magorrian, J., et al. 1998, AJ, 115, 2285

Magorrian, J. \& Tremaine, S. 1999, MNRAS, 309, 447

Maoz, D., Nagar, M. N., Falcke, H., \& Wilson, A. S. 2005, ApJ, 625, 699

Merritt, D. \& Ferrarese, L. 2001, MNRAS, 320, 30

Ramirez-Ruiz, E. \& Rosswog, S. 2008, ApJ, 697, L77

Rees, M. J. 1988, Nature, 333, 523

Renzini, A., et al. 1995, Nature, 378, 39

Ulmer, A. 1999, ApJ, 514, 180

Ulmer, A., Paczynski, B., \& Goodman, J. 1998, A\&A, 333, 379

Wang, J. \& Merritt, D. 2004, ApJ, 600, 149 